frequent in those who died $(13 / 20-65 \%)$ than those that survived $(5 / 40-12.5 \% ; p<0.001)$. There was a non-significant trend for DNAR to be less frequent in those $\leq 75(6 / 31-33 \%)$ than those aged $>75(12 / 29-67 \% ; p=0.091)$ and they were more frequent in those admitted from nursing homes $(5 / 7-71.4 \%)$ than from their own home $(9 / 49$ - 18.4\%; $p<0.001) .11 / 60(18.3 \%)$ were admitted to ICU but patients with DNAR were no more or less likely to be so managed $(5 / 18-27.8 \%$ cf $6 / 42-14.3 \% ; p=0.279)$. There was a trend for DNAR to have been recorded more often in the more severely ill. Rates by CURB65 score were $0-1 / 5(20 \%), 1-2 / 17$ (11.8\%), 2 - 3/15 (20\%), 3 - 8/17 (47.1\%), 4 - 3/5 (60\%), 5 - 1/1 $(100 \%) ; p=0.063$.

The high frequency of DNAR orders suggests that pneumonia deaths may not be as preventable as might be considered at first sight. This may be especially true for those aged $>75$. In any assessment of the predictability of death the use of DNAR orders should be considered.

\section{P17 THE ACCURACY OF A DIAGNOSIS OF PNEUMONIA IN A UK TEACHING HOSPITAL}

doi:10.1136/thoraxjnl-2012-202678.158

KL Pink, I Mitchell, HE Davies. University Hospital Llandough, Cardiff, UK

Background Obtaining an accurate diagnosis of pneumonia is an essential part of optimal patient care. Analysis of patients' hospital records allows clinical coding (ICD-10) of admission events which assist development of clinical decision algorithms, assessment of quality of care and public health evaluation. We sought to evaluate the reliability of applied clinical codes and the accuracy of a diagnosis of pneumonia in our institution.

Methods A retrospective case note review of all patients admitted to University Hospital Llandough in 2011 with a final clinical code diagnosis of pneumonia. Pneumonia was defined as the presence of new radiographic infiltrate in patients with symptoms consistent with an acute lower respiratory tract infection. ${ }^{1}$ The chest radiographs of each patient were reviewed by a respiratory physician (KP, HED) and the formal radiology report was independently scrutinised (IM)

Results 710 patient episodes of ICD-10 coded pneumonia were identified in a 1 year period at our hospital. Ten patients had no chest x-ray performed and one x-ray had no report. Radiological confirmation of pneumonia (by radiology reporting) occurred in $69.8 \%$ (488/699); a radiological diagnosis of pneumonia was made by a respiratory physician (KP, HED) in $71.8 \%(502 / 699)$ of patients.

There was $85 \%$ agreement between the Respiratory and Radiology reports (592/699 cases) with a kappa of 0.66 (95\% CI 0.57 to 0.69$)$.

The accuracy of a pneumonia diagnosis differed little between patients cared for by a respiratory physician (72.3\% agreement with radiology report) and those admitted to a non-respiratory ward (68.1\%). In $27.0 \%$ and $31.9 \%$ of patients respectively there was no radiological evidence of pneumonia.

Conclusions A clinical coding diagnosis of pneumonia is unreliable with $30.2 \%$ of patients not having compatible radiograph change. This has implications for the validity of any research performed on data selected on the basis of clinically coded information. Misdiagnosis by clinicians is the most likely reason for this discrepancy.

Reference

1. BTS Guidelines for the Management of Community Acquired Pneumonia in Adults Update 2009.

\section{P18 COMMUNITY ACQUIRED PNEUMONIA: IS MEDICAL ASSESSMENT UNIT SAFE AFTER HOURS?}

doi:10.1136/thoraxinl-2012-202678.159

A Khanna, P Sierra Perez, H Moxon, ML Billington, R Berg Derby. Royal Hospital NHS Trust, Derby, UK
Introduction Early radiological diagnosis is an important quality of care indicator in community acquired pneumonia (CAP), with evidence for negative impact of time to X-ray (TXR) $>4$ hours on length of stay and time to antibiotic administration ${ }^{1}$. Despite growing concern about impact of out-of-hours admission on outcomes in a variety of acute medical conditions, there is little information on impact of time of day on processes of care in CAP in the UK. We analysed impact on TXR of out-of-hour's admission via Emergency department (ED) versus Medical assessment unit (MAU) in a 1000 bed teaching hospital.

Methods Retrospective review of 300 consecutive adult admissions with radiologically confirmed CAP within a 3-month period. Data included point of entry to hospital, in-hours (08h00-16h00) versus out-of-hours admission, urgency of request, and time taken to order and perform CXR.

Results 210 patients (70\%) were admitted via ED and 90 (30\%) via MAU. Average TXR (TXR-Ave) overall was $3.20 \mathrm{hrs}$ and $80 \%$ had TXR $<4$ hours. $72 \%$ of ED's CXR requests were urgent vs. $56 \%$ in MAU $(p=0.3)$. Daytime TXR-Ave in ED was significantly shorter than MAU (2.20 hrs vs. $3.30 \mathrm{hrs} ; \mathrm{p}=0.0003)$. TXR-Ave in ED was 2.30 hours overall and was not significantly affected by admission out of hours. In contrast, after-hours admission via MAU was associated with significantly increased TXR-Ave (6.20hrs out-of- hours vs. 3.30 hrs in-hours; $p=0.0001)$, and TXR $>4$ hours $(58 \%$ vs. $25 \%$; $p=0.0025)$. Time from request to performance of CXR was not significantly different in vs. out-of- hours, however average time from admission to requesting CXR in MAU was significantly longer out of hours vs. in-hours (4.57hrs vs. $2.03 \mathrm{hrs}$; $\mathrm{p}=0.0001$ ).

Conclusions After- hours admission via MAU is associated with a significant increase in diagnostic delay in patients with CAP, largely attributable to delayed CXR requests. This may reflect delayed clerking due to reduced staffing after hours. Organisational and staffing factors associated with 4 hour ED trolley wait pressure may account for swifter and more consistent processes of care in ED. Further studies are required.

Reference

1. Bewick T et al; Clin Med. 2010 Dec; 10(6):563-7.

P19 VITAMIN D IN THE PREVENTION OF ACUTE RESPIRATORY

doi:10.1136/thoraxjnl-2012-202678.160

DA Jolliffe, AR Martineau, CJ Griffiths. Barts and The London School of Medicine and Dentistry, London, England

Introduction and Objectives Acute respiratory infections (ARI) cause significant morbidity and mortality: in the UK, during 2004, 33,957 deaths occurred due to pneumonia alone. Vitamin D metabolites enhance immunity to a wide range of respiratory pathogens in vitro, and numerous clinical studies have investigated whether vitamin $\mathrm{D}$ deficiency is a risk factor for ARI, or whether vitamin D supplementation prevents ARI. Systematic reviews of this literature are lacking, however. Our objective was to conduct a systematic review of clinical studies investigating the relationship between vitamin $\mathrm{D}$ status or the effect of vitamin D supplementation on risk of ARI.

Methods The PubMed database was searched on $7^{\text {th }}$ June 2012 using the terms 'vitamin D' and' respiratory infection'. Crosssectional studies, case-control studies, cohort studies or clinical trials in human subjects investigating the relationship between serum concentration of vitamin $\mathrm{D}$ metabolites or the effect of vitamin $\mathrm{D}$ supplementation on risk of ARI were included; ARI was defined as any infection of the respiratory tract with symptom duration of 30days or less. Studies relating exclusively to tuberculosis were excluded, as this is classically regarded as a chronic respiratory tract infection, with symptom duration usually exceeding 30 days.

Results Thirty-one studies reporting data from a total of 43,272 participants were included in our review. Of these, 19 were 
observational studies ( 3 cross-sectional, 8 case-control and 8 cohort) and 12 were randomised controlled trials. Sixteen of the 19 observational studies reviewed reported statistically significant associations between vitamin $\mathrm{D}$ deficiency and susceptibility to ARI, and 3 reported no such association. Six of the 12 clinical trials reviewed reported protective effects of vitamin $\mathrm{D}$ against ARI, while five reported null effects, and one reported an adverse effect on pneumonia recurrence.

Conclusions Observational studies report consistent associations between vitamin $\mathrm{D}$ deficiency and susceptibility to ARI in a wide range of age-groups in diverse clinical settings. By contrast, randomised controlled trials of vitamin $\mathrm{D}$ supplementation for the prevention of ARI report conflicting results, possibly reflecting varying prevalence of vitamin $\mathrm{D}$ deficiency in study populations and/or heterogeneity in vitamin $\mathrm{D}$ supplementation regimens investigated.

\section{P20 FATIGUE AND POOR LUNG FUNCTION ARE SIGNIFICANTLY ASSOCIATED WITH IMPAIRED HEALTH-RELATED QUALITY OF LIFE (HROOL) IN A LARGE COHORT OF PATIENTS WITH CHRONIC PULMONARY ASPERGILLOSIS}

doi:10.1136/thoraxjnl-2012-202678.161

KA Al-shair, GT Atherton, DK Kennedy, GP Powell, DWD Denning. The National Aspergillosis Centre, University Hospital of South Manchester, Manchester University, Manchester, UK

Introduction Fatigue is a prominent disabling symptom in several chronic pulmonary diseases; however, its impact on HRQoL in patients with chronic pulmonary aspergillosis (CPA) has not been investigated.

Method Our 154 patients with CPA completed the Manchester COPD Fatigue Scale (MCFS), Thorax 2009)) and the SGRQ in our specialist referral centre. MCFS measures total fatigue and sub-components comprehensively. Lung function and body mass index were measured. Univariate and multivariate linear and binary analysis, and the principal component analysis (PCA) were used.

Results The mean (SD) age $(61.1$ (10.8)) years and 44\% were female; $\mathrm{FEV}_{1} \%$ (63.3 (24.9)), BMI (23.7 (5.2)), SGRQ total score (55.6 (23.5) and MCFS total score (30 (14.9)).

PCA showed that 27 items of MCFS loaded clearly on three components: physical and psychosocial and cognitive fatigue.

Univariate analysis showed a strong association between total SGRQ score and MCFS score $(r=0.81, p<0.001)$. Using total SGRO as a dependent variable, linear multi-variate analysis showed that fatigue was the strongest factor (beta $=0.83 p<0.0001$ ) associated with impaired health status followed by $\mathrm{FEV}_{1} \%$ (beta $=-0.22$, $p=0.009)$, but no statistically significant association with age, BMI and pack/years. This model explained $70 \%$ of the variance of SGRO total score.

Using patients' self-assessment grades of SGRQ (Very poor, poor, fair, good and very good), one-way ANOVA showed that patients with "very poor" health status had the highest fatigue scores (45 (6.4)), following by poor (35 (10.1)), fair (30 (10.4)), good (14 (10.9)) and very good $(0)(p<0.001)$. Splitting the group to (very poor and poor) versus (fair, good and very good), the ROC curve analysis indicated significant ability of MCFS and its components to detect change in HS (AUC $=0.82$; range $0.75-0.9, \mathrm{p}<0.0001$ ) as demonstrated in figure 1.

Furthermore, binary regression analysis showed that only fatigue score $(\mathrm{OR}=0.92,95 \% \mathrm{CI} 0.87-0.97 ; \mathrm{p}=0.002)$ and $\mathrm{FEV}_{1} \%(\mathrm{OR}=1.04$, $95 \%$ CI 1.01-1.07, $\mathrm{p}=0.02)$ are significantly associated with impaired health status after correcting to age, gender and DLCO\%. Conclusion This is the first study directly implicating fatigue as a major factor affecting health-related-quality of life in patients with CPA.
Figure 1, ROC curve analysis of the MCFS and its components; discrimination between health status categories

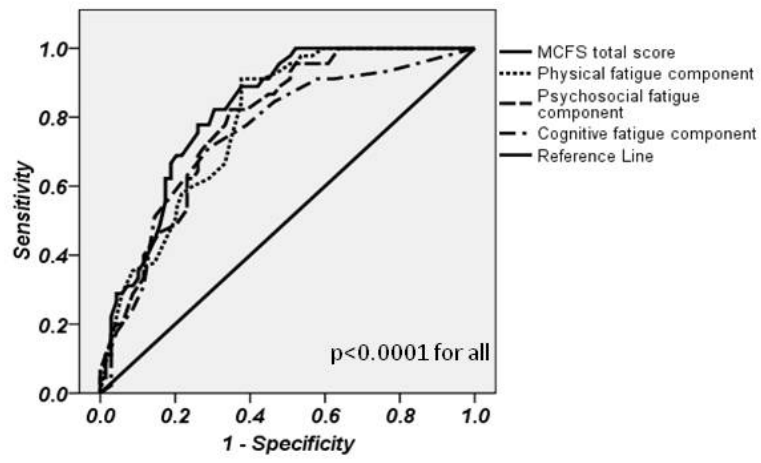

Abstract P20 Figure 1

\section{P21 LONG TERM ANTIFUNGAL TREATMENT (LTAFT) IS EFFECTIVELY ASSOCIATED WITH IMPROVEMENT IN HEALTH STATUS (HS) IN PATIENTS WITH CHRONIC PULMONARY ASPERGILLOSIS (CPA)}

doi:10.1136/thoraxjnl-2012-202678.162

KA Al-shair, MK Kirwan, GA Atherton, AC Caress, DWD Denning. The National Aspergillosis Centre, University Hospital of South Manchester, Manchester University, Manchester, UK

Introduction $\mathrm{CPA}$ is a chronic progressive respiratory infectious disease results in significant lung tissue destruction with a $50 \%+5$ year mortality. Response to antifungal therapy is slow, with $\sim 80 \%$ of patients who respond doing so by 6 months. We recently demonstrated the reliability and validity of SGRQ in examining HS in CPA (Chest, in press), and now present longitudinal data on the efficacy of LTAFT in improving HS in CPA patients.

Method HS of 98 CPA patients were assessed 3 times over 6 months using the well-established standardised SGRO. CPA severity was assessed using our published CPA banding system. $\mathrm{FEV}_{1}$, BMI, dyspnoea (using MRC dyspnoea scale) were measured.

Results Mean age was 58 years and 48\% were female; and 25, 58 and 15 had band 1, 2 and 3 CPA respectively. At visit 2 and 3 (V2 and V3), we found that overall total and domain SGRO scores were either lower (improved health status) or similar compared to V1 (table 1).

Categorizing the cohort by those who reported improvement or deterioration by a total SGRO score of $\geq 4$ at V3 comparing to V1, we found that $43 \%$ improved, $22 \%$ remained stable and $35 \%$ deteriorated. The median (IOR) of total SGRQ score of the improved group at V3 was 58 (42-66) compared to 71 (60-79) at V1; and for the deteriorated group was $67.5(57-76)$ at V3 compared to 62 $(41-67))$ at $\mathrm{V} 1$. The deteriorated were older (62 (9.8) years versus 56.1 (9.3) ( $p=0.008)$; and tended to have lower BMI, more dyspnoea and worse lung function. Moreover, binary regression multivariate analysis showed that age maintained its association with deterioration in HS (OR 1.13, 95\% CI 1.01-1.26, $\mathrm{p}=0.03$ ) after correcting for gender, $\mathrm{BMI}$ and $\mathrm{FEV}_{1} \%$.

Of the 37 patients started on an antifungal agent at V1 who took it for $3+$ months (including a 3 week IV course of amphotericin B), $22(59 \%)$ improved, 11 (30\%) were stable and 4 (11\%) deteriorated at V3.

Conclusion LTAFT prevented/reduced the progression of CPA and patients preserved overall good HS. More therapeutic approaches for this progressive disease are urgently needed. 